
\title{
25 Research Square \\ Safety and Feasibility of Umbilical Cord Mesenchymal Stem Cells in Patients with COVID-19 Pneumonia: A Pilot Study
}

\section{Ying Feng}

Wuhan University Zhongnan Hospital

Jianying Huang

Wuhan University Zhongnan Hospital

Jianyuan Wu

Wuhan University Zhongnan Hospital

Yan Xu

Jilin Tuohua Biotechnology Co.,Ltd.

\section{Bo Chen}

Wuhan University Zhongnan Hospital

\section{Lijun Jiang}

Jilin Tuohua Biotechnology Co.,Ltd.

\section{Hui Xiang}

Wuhan University Zhongnan Hospital

\section{Zhiyong Peng}

Wuhan University Zhongnan Hospital

Xinghuan Wang ( $\nabla$ wangxinghuan@whu.edu.cn)

Wuhan University Zhongnan Hospital

\section{Research}

Keywords: Coronavirus disease 2019, Umbilical cord mesenchymal stem cells, Cytokine storm, Feasibility

Posted Date: August 21st, 2020

DOl: https://doi.org/10.21203/rs.3.rs-62218/v1

License: (c) (1) This work is licensed under a Creative Commons Attribution 4.0 International License. Read Full License

Version of Record: A version of this preprint was published at Cell Proliferation on November 17th, 2020. See the published version at https://doi.org/10.1111/cpr.12947. 


\section{Abstract}

Background: We aim to explore the safety and feasibility of umbilical cord mesenchymal stem cells (UCMSCs) transplantation in severe and critically severe type Coronavirus disease 2019 (COVID-19) patients.

Methods: In addition to normal therapy, we performed four times transplantation of UC-MSCs in 16 severe and critically severe type COVID-19 patients. We observed adverse events from enrollment to D28. We evaluated the oxygenation index, inflammatory biomarkers, chest imaging, lymphocyte subsets count et al on the 7 th day (D7 \pm 1 day), the 14 th day (D14 \pm 1 day) and the 28 th day (D28 \pm 3 days).

Results: There were no infusion-related or allergic reactions. The oxygenation index was improved after transplantation. The mortality of enrolled patients was $6.25 \%$. The level of cytokines estimated was varied in normal range, the chest imaging was improved, the lymphocyte count and lymphocyte subsets count were recovered after transplantation.

Conclusions: Intravenous transplantation of UC-MSCs was safe and feasible for treatment in patients with COVID-19 pneumonia.

Trial registration: Clinical Trial, NCT04269525. Registered 7 February 2020. https://clinicaltrials.gov/ct2/show/NCT04269525

\section{Background}

Since the first case reported in December 2019 in Wuhan, novel coronavirus disease 2019 (COVID-19) has grown to be a global public health emergency. Till now, the total infected number has reached $10,000,000$ with more than 500,000 deaths in 216 countries, areas or territories. It is important to find a safe and effective treatment for this COVID-19 besides controlling the pandemic. Although clinicians and researchers have tried their best to find a solution, there is no specific cure presently for COVID-19 to date.

As the virus can cause a terrible cytokine storm in the lung, such as Interleukin-2 (IL-2), Interleukin-6 (IL-6), Interleukin-7 (IL-7), interferon-induced protein 10 (IP10), monocyte chemoattractant protein 1 (MCP1), macrophage inflammatory protein $1 \mathrm{a}$ (MIP1a), and tumor necrosis factor-a (TNF-a), followed by edema, dysfunction of the air exchange, acute respiratory distress syndrome (ARDS), acute cardiac injury, secondary infection, leading to sepsis and multiorgan failure, which may lead to death[1], any treatment that contributes to inhibit the terrible cytokine storm will represent a major step forward.

Under this situation, stem cell therapy has become a promising therapeutic strategy due to their potential of self-renewal, multidirectional differentiation, anti-inflammatory and immune regulatory functions. Stem cells can be attracted to the site of injury to contribute to organ repair and have the capability to foster endogenous progenitor cell function in the lung[2]. Mesenchymal stem cells (MSCs) do not trigger a host response or cell rejection response due to their insensitivity to pro-inflammatory interferon- $\gamma$ (IFN- $\gamma$ ) induced human leukocyte antigen-II (HLA-II) expression[3], making them safer than other kinds of stem 
cells. Leng et al has reported that MSC transplantation improves the outcome of 7 enrolled patients with COVID-19 pneumonia in Beijing[4]. After intravenous infusion, MSCs accumulate in the lung, which could potentially improve the pulmonary microenvironment, protect alveolar epithelial cells, prevent pulmonary fibrosis and improve lung function[5]. In the meanwhile, MSCs can secrete many types of cytokines by paracrine secretion or make direct interactions with immune cells leading to immunomodulation.

Here we conducted a pilot study to evaluate the feasibility and safety of intravenous infusion of umbilical cord MSCs (UC-MSCs) in severe and critically severe COVID-19 patients.

\section{Methods}

\subsection{Study design}

A pilot trial of intravenous infusion of UC-MSCs in 16 severe and critically severe COVID-19 patients were conducted in Zhongnan Hosiptal of Wuhan University and Wuhan Leishenshan Hospital, Wuhan, Hubei, China, and approved by the ethics committee of the hospital (Clinical Ethical Approval NO. 202002). We also registered in Clinical Trials (NCT 04269525).

\subsection{Patients}

Patients were enrolled from Feb 7th to April 1st, 2020. 16 patients were enrolled, and a flow diagram is showed in Fig. 1. In our protocol, the follow up time would be 28 days in total. Due to the quarantine, the actual follow up time was 39 days (7-67 days). Inclusion criteria were the following: 1) 18-80 years of age; 2 ) diagnosed as severe or critically severe COVID-19 according to guidance of National Health Commission of China (diagnostic criteria shown in Table 1); 3 ) a positive reverse-transcriptasepolymerase-chain-reaction assay (DAAN Gene Co., Ltd) for severe acute respiratory syndrome coronavirus 2 (SARS-CoV-2) in a respiratory tract sample or a positive antibody assay (Vazyme Biotech Co., Ltd) in blood tested by a designated diagnostic laboratory; 4) Male or nonpregnant female who were willing to use effective contraception during the trial, women of childbearing potential (ie, not post-menopausal or surgically sterilized) must have a negative serum pregnancy test before randomization; 5) A signed informed consent form. If a study patient is unable to provide informed consent due to their medical condition, the patient's legally authorized representative may consent on behalf of the study patient, as permitted by local law and institutional Standard Operating Procedures. Exclusion criteria were the following: 1) Sequential Organ Failure Assessment(SOFA)score for liver over 3 points; 2) positive for human immunodeficiency virus (HIV) antibody; 3 ) history of significant hypersensitivity or allergic reaction; 4) pregnant and lactating women; 5) malignant tumor patients; 6) patients who had pulmonary embolism; 7) participation in a clinical study within 3 months; 8) Unable or unwilling, in the opinion of the Investigator, to comply with the protocol. 
Table 1

Clinical classification of the COVID-19 released by the National Health Commission of China.

\section{Severe}

Meet any of the followings:

1. Respiratory distress, $\mathrm{RR} \geq 30 / \mathrm{min}$;

2. Oxygen saturation $\leq 93 \%$ at rest state;

3. Oxygenation index $\leq 300 \mathrm{mmHg}$, $1 \mathrm{mmHg}=0.133 \mathrm{kPa}$

\section{Critically severe}

Meet any of the following:

1. Respiratory failure needs mechanical ventilation;

2. Shock;

3. Combined with other organ failure, patients need ICU monitoring and treatment.

\subsection{Cell preparation and transplantation}

The clinical grade UC-MSCs were supplied, for free, by Jilin Tuohua Biotechnology Co., Ltd. (Changchun, Jilin, 130000, China). The cell product has been certified by National Institutes for Food and Drug Control (Report number: SH201301098, SH201301175, SH201301317, SH201500350, SH201500351, SH201500477, SH201701982, SH201701983). Before the intravenous drip, UC-MSCs were suspended in $50 \mathrm{ml}$ of normal saline, and the total number of transplanted cells was $1 \times 10^{8}$ cells once. The patients would receive four times transplantation in total every other day. The transplantation was performed about 1.5 hours with a speed of $30 \sim 60$ drops per minute.

\subsection{Measurements}

Patients assessed at the time of enrollment for basic physical parameters, $\beta$-human chorionic gonadotropin (for females of childbearing age), COVID-19 clinical classification, SARS-CoV-2 nucleic acid (nasal swap) and antibody, HIV antibody, $\mathrm{PaO}_{2}$, blood routine examination, blood biochemistry, urinalysis, coagulation function, inflammatory biomarkers (white blood cell (WBC) count, lymphocyte count, IL-2, Interleukin-4 (IL-4), IL-6, Interleukin-10 (IL-10), TNF-a, IFN-y, procalcitonin (PCT) and C reactive protein (CRP)), myocardial enzymes, lymphocyte subsets (CD4 ${ }^{+}$T-cells, $\mathrm{CD} 8^{+}$T-cells and natural killer (NK) cells), electrocardiograph and chest imaging (X-ray or CT).

After enrollment, basic physical parameters were recorded every day. We also assessed the SARS-CoV-2 nucleic acid (nasal swap) and antibody, $\mathrm{PaO}_{2}$, blood routine examination, blood biochemistry, urinalysis, coagulation function, inflammatory biomarkers (WBC count, lymphocyte count, IL-2, IL-4, IL-6, IL-10, TNFa, IFN- $\gamma$, PCT and CRP), myocardial enzymes, lymphocyte subsets count (CD4 ${ }^{+} \mathrm{T}$-cells, CD8 ${ }^{+} \mathrm{T}$-cells and NK cells), electrocardiograph and chest imaging (X-ray or CT) on the 7th day (D7 \pm 1 day), the 14th day (D14 \pm 1 day) and the 28th day (D28 \pm 3 day). Adverse events and concomitant medication were collected.

\subsection{Outcome definitions}

The primary outcome was oxygenation index on D14. The secondary outcomes were: 1) mortality on D28; 2) total length of hospital stay; 3) SARS-CoV-2 nucleic acid or antibody assay on D7, D14 and D28; 
4) chest imaging on D7, D14 and D28; 5) inflammatory biomarkers on D7, D14 and D28; 6) lymphocyte subsets count on D7, D14 and D28.

\subsection{Statistical analysis}

All data were collected through EpiData and then imported into the SAS 9.4 statistical package. Figures were performed with GraphPad.v8 (GraphPad software, San Diego). Categorical variables were described by frequency and proportions. Continuous variables were described by mean values when normally distributed or median and range when skewed distributed. The variables were tested using Fisher exact or Student $t$ test, as appropriated. All descriptive statistical analysis was performed with SAS 9.4 (SAS Institute, Inc., Cary, NC). All p values were 2 -sided, and a p value less than 0.05 was considered statistically significant.

\section{Results}

\subsection{Patients characteristics}

A total of 16 patients with COVID-19 were enrolled and all finished UC-MSCs transplantation four times on D1, D3, D5 and D7, of which 9 patients were severe type (Group A) and 7 patients were critically severe type (Group B). The demographic information of the 16 patients is listed in Table 2. For the small sample size of patients in each group, we just used a descriptive analysis.

Table 2

Demographic characteristics of enrolled patients

\begin{tabular}{|llll|}
\hline & Group A & Group B & Total \\
\hline $\mathrm{N}$ & 9 & 7 & 16 \\
\hline Age, mean (SD), y & $62.33(11.29)$ & $61.00(8.94)$ & $61.75(10.02)$ \\
\hline Male & $6(66.67 \%)$ & $6(85.71 \%)$ & $12(75.00 \%)$ \\
\hline Comorbidities & & & \\
\hline Hypertension & $2(22.22 \%)$ & $6(85.71 \%)$ & $8(50.00 \%)$ \\
\hline Diabetes & $2(22.22 \%)$ & $4(57.14 \%)$ & $6(37.50 \%)$ \\
\hline Chronic kidney failure & 0 & $3(42.86 \%)$ & $3(18.75 \%)$ \\
\hline Hepatitis B & $1(11.11 \%)$ & 0 & $1(6.25 \%)$ \\
\hline Bronchial asthma & $1(11.11 \%)$ & 0 & $1(6.25 \%)$ \\
\hline Alzheimer's disease & $1(11.11 \%)$ & 0 & $1(6.25 \%)$ \\
\hline Anemia & $1(11.11 \%)$ & 0 & $1(6.25 \%)$ \\
\hline
\end{tabular}


No acute infusion-related or allergic reactions were observed within two hours after transplantation. Similarly, no delayed hypersensitivity or secondary infections due to UC-MSCs transplantation were detected after treatment. There were two severe adverse events (SAE) during the trial, both of the patients were suffered from bacterial pneumonia and septic shock, leading to multiorgan functional failure and death. The two SAEs were considered to have no relationship with UC-MSCs transplantation. The first SAE was from a male patient, diagnosed as critical severe type COVID-19 pneumonia with hypertension and diabetes, and accepted three times transplantation of UC-MSCs. The patient worsened on D3 due to bacterial infection with elevated white blood cell count and PCT. He developed multiorgan function failure on D5 and died on D6. The bacterial culture of UC-MSCs was negative. The second SAE was from a female patient, diagnosed as critical severe type COVID-19 pneumonia with Alzheimer's disease, and finished four times transplantation of UC-MSCs. The patient worsened on D13 due to aspiration. We upgraded antibiotics and enhanced airway care. However, the patient didn't improve after the treatment and developed ventricular fibrillation on D19. After resuscitation, she restored spontaneous circulation, but the infection was difficult to control. Although we tried our best, the patient died on D23 due to circulation and respiratory failure. Other adverse events included hypoproteinemia, sleeplessness, gastrointestinal disease, paroxysmal arrhythmia. The transplantation was through intravenous infusion without any special equipment.

\subsection{The primary outcome}

The primary outcome was the oxygenation index on D14. Before UC-MSCs transplantation, the oxygenation index in group $A(n=8)$ and group $B(n=7)$ were $285.50(197.50-469.00) \mathrm{mmHg}$ and 177.14 $(92.50-316.00) \mathrm{mmHg}$, respectively with the mean oxygenation index in total $258.80(92.50-469.00)$ $\mathrm{mmHg}$. After four times transplantation, the oxygenation index increased into 329.00 (197.70-604.00) $\mathrm{mmHg}$ and $316.84(93.30-531.00) \mathrm{mmHg}$ in group $\mathrm{A}(\mathrm{n}=9)$ and group $\mathrm{B}(\mathrm{n}=6)$ with the oxygenation index in total 325.70 (93.30-604.00) $\mathrm{mmHg}$ on D7. However, 5 patients in group $A$ and 3 patients in group B missed the arterial blood gas analysis on D14. The oxygenation index was 356.95 (107.50452.40) $\mathrm{mmHg}$ and $453.79(306.00-552.30) \mathrm{mmHg}$ in group $A(n=4)$ and group $B(n=4)$ with the oxygenation index in total 394.79 (107.50-552.30) $\mathrm{mmHg}$ on D14. Despite the small number of enrolled patients, the oxygenation index was improved after UC-MSCs transplantation (Fig. 2).

\subsection{The secondary outcome}

The mortality on D28 in total was $6.25 \% .1$ patient died in group $A$ and 1 patient died in group $B$, there was no statistical significance between severe type and critically severe type $(P=1.0000)$. The SARS-CoV2 nucleic acid and antibody assay results were shown in Table 3 and Table 4 . The chest imaging results were reported by independent radiologist and all showed improvement compared with baseline. The white blood cell count and lymphocyte count were shown in Fig. 3. The lymphocyte subsets count was shown in Fig. 4. The inflammatory biomarkers were shown in Fig. 5 and Fig. 6. 
Table 3

SARS-CoV-2 nucleic acid assay

\begin{tabular}{|lllll|}
\hline & & Group A & Group B & Total \\
\hline Baseline & $\mathrm{N}$ (missing) & $9(0)$ & $7(0)$ & $16(0)$ \\
\hline \multirow{2}{*}{ D7 } & Positive, n (\%) & $1(11.11)$ & $2(28.57)$ & $3(18.75)$ \\
\hline & $\mathrm{N}$ (missing) & $7(2)$ & $5(2)$ & $12(4)$ \\
\hline D14 & Positive, n (\%) & $0(0.00)$ & $1(20.00)$ & $1(8.33)$ \\
\hline \multirow{2}{*}{ D28 } & N (missing) & $4(5)$ & $4(3)$ & $8(8)$ \\
\hline & Positive, n (\%) & $1(25.00)$ & $0(0.00)$ & $1(12.50)$ \\
& N (missing) & $5(4)$ & $4(3)$ & $9(7)$ \\
\hline
\end{tabular}

Table 4

SARS-CoV-2 antibody assay

\begin{tabular}{|c|c|c|c|c|c|}
\hline & & & Group A & Group B & Total \\
\hline \multirow[t]{3}{*}{ Baseline } & & $\mathrm{N}$ (missing) & $8(1)$ & $4(3)$ & $12(4)$ \\
\hline & $\lg M$ & Positive, n (\%) & $6(75.00)$ & $2(50.00)$ & $8(66.67)$ \\
\hline & $\lg G$ & Positive, n (\%) & $8(100.00)$ & $4(100.00)$ & $11(100.00)$ \\
\hline \multirow[t]{3}{*}{ D7 } & & $\mathrm{N}$ (missing) & $6(3)$ & $3(4)$ & $9(7)$ \\
\hline & $\lg M$ & Positive, n (\%) & $5(83.33)$ & $2(66.67)$ & $7(77.78)$ \\
\hline & $\lg G$ & Positive, n (\%) & $6(100.00)$ & $3(100.00)$ & $9(100.00)$ \\
\hline \multirow[t]{3}{*}{ D14 } & & $\mathrm{N}$ (missing) & $1(8)$ & $2(5)$ & $3(13)$ \\
\hline & $\lg M$ & Positive, n (\%) & $0(0.00)$ & $0(0.00)$ & $0(0.00)$ \\
\hline & $\lg G$ & Positive, n (\%) & $1(100.00)$ & $0(0.00)$ & $1(33.33)$ \\
\hline \multirow[t]{3}{*}{ D28 } & & $\mathrm{N}$ (missing) & $4(5)$ & $2(5)$ & $6(10)$ \\
\hline & $\lg M$ & Positive, n (\%) & $2(50.00)$ & $0(0.00)$ & $2(33.33)$ \\
\hline & $\lg G$ & Positive, n (\%) & $4(100.00)$ & $1(50.00)$ & $5(83.33)$ \\
\hline
\end{tabular}

\section{Discussion}

We conducted a pilot trial of intravenous infusion of UC-MSCs in 16 severe and critically severe COVID-19 patients, confirming its safety and feasibility, with a significant increase in oxygenation index and 
relatively low mortality. The improvement of chest imaging, recovery of lymphocyte count and decrease of cytokine level were also observed in our trial, making UC-MSCs transplantation a promising treating strategy.

Safety of MSC transplantation has been identified in previous clinical trials treating ARDS[6, 7]. In the recent studies about MSC transplantation in COVID-19 patients, there was no MSC related adverse event either. In our study, there was no acute infusion-related or allergic reactions were observed, and no delayed hypersensitivity or secondary infections. As MSCs trend to accumulate in pulmonary circulation after infusion, the pulmonary embolism risk increased rapidly. In our study, none of the patients developed a thromboembolic event.

Multiple clinical trials using stem cell therapy to treat the COVID-19 have been registered at www.clinicaltrials.gov. Two published studies showed that ACE2 ${ }^{-}$MSC and exosomes derived from bone marrow MSC could improve the clinical outcome of COVID-19 patients[4, 8]. In accordance with our findings, the use of exosomes derived from bone marrow MSC could increase the oxygenation index of severe COVID-19 patients which may reduce the need for invasive ventilator and shorten hospital stay[8].

The outcome of COVID-19 patients admitted to the ICU is poor. In a recent series of 1581 Italian patients in Lombardy Region with COVID-19 ARDS admitted to ICU, the mortality was $26 \%$ and only $16 \%$ had been discharged[9]. In another series of patients in Milan, Italy, the mortality was $23 \%$ and $31 \%$ had been discharged[10]. In Vancouver, Canada, the mortality in ICU patients was 15.4\%[11]. In our study, the mortality was about $6.25 \%$ which was relatively lower.

It is noteworthy that adults with COVID-19 often present with a profound decrease in lymphocyte count including $\mathrm{CD}^{+}$and $\mathrm{CD} 8^{+}$T-cell subsets at the early stage of this disease[12-14]. Qin et al also reported that severe cases of COVID-19 were likely to have lower lymphocyte count compared with non-severe patients[14]. More recently, $C D 8^{+}$T-cells have been reported to be significantly decreased in peripheral blood in patients with COVID-19[15]. More importantly, CD8 ${ }^{+} \mathrm{T}$-cells $\leq 75$ cells $/ \mu \mathrm{L}$ was a reliable predictor for mortality of patients with COVID-19[16]. In our study, patients in group A had higher lymphocyte count than group B. Although patients enrolled in Leishenshan Hospital were not able to take the lymphocyte subsets test, the patients survived had $\mathrm{CD} 8^{+} \mathrm{T}$-cells all over $75 \mathrm{cells} / \mu \mathrm{L}$, while the non-survival patient only had 18 cells $/ \mu \mathrm{L} \mathrm{CD} 8^{+}$T-cells at baseline. After the transplantation of UC-MSC, the lymphocyte count including $\mathrm{CD} 4^{+} \mathrm{T}$-cell subsets, $\mathrm{CD} 8^{+} \mathrm{T}$-cell subsets, and NK cells were increased, which suggested the immunomodulation effect of UC-MSC may play an important role in the COVID-19 treatment.

In a subset of COVID-19 patients who progress to pneumonia, respiratory failure and death by the end of the first week showed extreme rise in inflammatory cytokines including IL2, IL7, IL10, and TNF-a[17]. High levels of expression of IL-1 $\beta$, IFN- $\gamma$, IP-10 have been detected in patients with COVID-19 [1]. The serum levels of IL-2R and IL-6 in patients with COVID-19 are positively correlated with the severity of the disease[18]. In our study, cytokines include IL-2, Interleukin-4, IL-6, IL-10, IFN-y, and TNF-a were tested. As we accepted the results 7 days before enrollment, the baseline cytokines were relatively low, which might 
not in accordance with the real clinical situation. MSC could inhibit the secretion of pro-inflammatory cytokines, such as, IL-1, TNF-a, IL-6, Interleukin-12, and IFN-ץ, thereby reducing the occurrence of cytokine storms $[19,20]$. Meanwhile, MSC can secrete Interleukin-10, hepatocyte growth factor, keratinocyte growth factor and vascular endothelial growth factor to alleviate ARDS, regenerate and repair damaged lung tissues, and resist fibrosis[21]. After the transplantation of UC-MSCs, the cytokine level varied in the normal range, which might prove the anti-inflammatory effect of UC-MSCs.

Our study has several limitations. First, the trial was lack of randomization, blinding, and comparison, with small sample size, which made it difficult to evaluate the efficacy of UC-MSC. Second, there was no specific statistics on the total length of hospital stay. During this pandemic in China, the COVID-19 patients were being treated in different designated hospitals in different disease period for treatment and quarantine. The hospital information systems were separate in different hospitals, made the total length of hospital stay beyond compute. Third, the lost rate of follow up and plan deviation, especially the missing of laboratory tests, were relatively higher than usual, due to the quarantine policy and psychological distress after the disease, made it difficult to do statistical analysis. Further high-quality randomization clinical trials are needed to provide more specific evidence.

\section{Conclusion}

In summary, our results indicate that UC-MSCs can be safely administered in critically ill patients with COVID-19 pneumonia and that administration of UC-MSCs is associated with clinical benefit and changes in inflammatory and immune populations. UC-MSCs have fast doubling times, they can be efficiently expanded in the lab, which makes it feasible to carry out randomization clinical trials.

\section{Abbreviations}

COVID-19

coronavirus disease 2019

IL-2

Interleukin-2

IL-6

Interleukin-6

IL-7

Interleukin-7

IP-10

interferon-induced protein 10

MCP1

monocyte chemoattractant protein 1

MIP1a

macrophage inflammatory protein $1 a$

TNF-a 
tumor necrosis factor-a

ARDS

acute respiratory distress syndrome

MSCs

mesenchymal stem cells

IFN-y

interferon- $\gamma$

HLA-II

human leukocyte antigen-II

UC-MSCs

umbilical cord mesenchymal stem cells

SARS-CoV-2

severe acute respiratory syndrome coronavirus 2

SOFA

Sequential Organ Failure Assessment

HIV

human immunodeficiency virus

IL-4

Interleukin-4

IL-10

Interleukin-10

PCT

procalcitonin

CRP

$C$ reactive protein

SAE

severe adverse event

\section{Declarations}

\section{Ethics approval and consent to participate}

Study protocols were approval by the local ethics committee of the Zhongnan Hospital of Wuhan University (Clinical Ethical Approval NO. 202002). Written informed consent was obtained before screening.

\section{Consent for publication}

Not applicable

\section{Availability of data and material}


The datasets used and/or analyzed during the current study are available from the corresponding author on reasonable request.

\section{Competing interests}

The authors declare that they have no competing interests.

\section{Funding}

The clinical trial was supported by the Special Project for Significant New Drug Research and Development in the Major National Science and Technology Projects of China (Project NO. 2020ZX09201007) and Emergency Science and Technology Research Project for COVID-19 of Hubei Province Science Technology Department (Project NO. 2020FCA048).

\section{Authors' contributions}

$\mathrm{ZP}, \mathrm{XW}$ conceived and designed the study. YF and HX performed the patient enrollment, patient intervention. YF and BC performed the follow-up. JW and BC completed statistical analysis. YX and LJ contributed umbilical cord mesenchymal stem cells' preparation. JH performed quality control of the trial. YF and JH wrote the paper. All authors read and approved the final manuscript.

\section{Acknowledgements}

We thank Professor Xiuli Zhao from Clinical Trial Center in Beijing Tongren Hospital, Professor Jinyu Liu from Jilin University, and Professor Qifa Ye from Zhongnan Hospital for their guidance in trial implementation. We also thank Lina Zhang and Na Tian from Jilin Tuohua Biotechnology Co., Ltd. for their contribution on UC-MSC preparation and coordination. We also would like to thank Professor Ping Yin from School of Public Health, Tongji Medical College, Huazhong University of Science and Technology for his help in data analysis. We also thank China Medicinal Biotechnology Association for their guidance in program registration in National Health Commission of China and National Medical Products Administration.

\section{References}

1. Huang C, Wang Y, Li X et al (2020) Clinical features of patients infected with 2019 novel coronavirus in Wuhan. China Lancet 395:497-506. DOI:10.1016/S0140-6736(20)30183-5

2. Tropea KA, Leder E, Aslam M et al (2012) Bronchioalveolar stem cells increase after mesenchymal stromal cell treatment in a mouse model of bronchopulmonary dysplasia. Am J Physiol Lung Cell Mol Physiol 302:L829-L837. DOI:10.1152/ajplung.00347.2011

3. Yang J, Jia Z (2014) Cell-based therapy in lung regenerative medicine. Regen Med Res 2:7. DOI:10.1186/2050-490X-2-7 
4. Leng Z, Zhu R, Hou W et al (2020) Transplantation of ACE2(-) Mesenchymal Stem Cells Improves the Outcome of Patients with COVID-19 Pneumonia. Aging Dis 11:216-228.

DOI:10.14336/AD.2020.0228

5. Behnke J, Kremer S, Shahzad T et al.(2020) MSC Based Therapies-New Perspectives for the Injured Lung. J Clin Med 9. DOI:10.3390/jcm9030682

6. Wilson JG, Liu KD, Zhuo H et al (2015) Mesenchymal stem (stromal) cells for treatment of ARDS: a phase 1 clinical trial. Lancet Respir Med 3:24-32. DOI:10.1016/S2213-2600(14)70291-7

7. Matthay MA, Calfee CS, Zhuo H et al (2019) Treatment with allogeneic mesenchymal stromal cells for moderate to severe acute respiratory distress syndrome (START study): a randomised phase 2a safety trial. Lancet Respir Med 7:154-162. DOI:10.1016/S2213-2600(18)30418-1

8. Sengupta V, Sengupta S, Lazo A et al (2020) Exosomes Derived from Bone Marrow Mesenchymal Stem Cells as Treatment for Severe COVID-19. Stem Cells Dev 29:747-754.

DOI:10.1089/scd.2020.0080

9. Grasselli G, Zangrillo A, Zanella A et al (2020) Baseline Characteristics and Outcomes of 1591 Patients Infected With SARS-CoV-2 Admitted to ICUs of the Lombardy Region. Italy JAMA. DOI:10.1001/jama.2020.5394

10. Zangrillo A, Beretta L, Scandroglio AM et al.(2020) Characteristics, treatment, outcomes and cause of death of invasively ventilated patients with COVID-19 ARDS in Milan, Italy. Crit Care Resusc

11. Mitra AR, Fergusson NA, Lloyd-Smith E et al.(2020) Baseline characteristics and outcomes of patients with COVID-19 admitted to intensive care units in Vancouver, Canada: a case series. CMAJ. DOI: $10.1503 / \mathrm{cmaj} .200794$

12. Zhou F, Yu T, Du R et al (2020) Clinical course and risk factors for mortality of adult inpatients with COVID-19 in Wuhan, China: a retrospective cohort study. Lancet 395:1054-1062. DOI:10.1016/S0140-6736(20)30566-3

13. Liu J, Liu Y, Xiang P et al (2020) Neutrophil-to-lymphocyte ratio predicts critical illness patients with 2019 coronavirus disease in the early stage. J Transl Med 18:206. DOI:10.1186/s12967-020-02374-0

14. Qin C, Zhou L, Hu Z et al.(2020) Dysregulation of immune response in patients with COVID-19 in Wuhan, China. Clin Infect Dis. DOI: 10.1093/cid/ciaa248

15. Liu Y, Yang Y, Zhang C et al (2020) Clinical and biochemical indexes from 2019-nCoV infected patients linked to viral loads and lung injury. Sci China Life Sci 63:364-374. DOI:10.1007/s11427020-1643-8

16. Du RH, Liang LR, Yang CQ et al (2020) Predictors of mortality for patients with COVID-19 pneumonia caused by SARS-CoV-2: a prospective cohort study. Eur Respir J 55. DOI:10.1183/13993003.005242020

17. Chen N, Zhou M, Dong X et al (2020) Epidemiological and clinical characteristics of 99 cases of 2019 novel coronavirus pneumonia in Wuhan, China: a descriptive study. Lancet 395:507-513. DOI:10.1016/S0140-6736(20)30211-7 
18. Chen L, Liu HG, Liu W et al (2020) [Analysis of clinical features of 29 patients with 2019 novel coronavirus pneumonia]. Zhonghua Jie He He Hu Xi Za Zhi 43:203-208.

DOI:10.3760/cma.j.issn.1001-0939.2020.03.013

19. Uccelli A, de Rosbo NK (2015) The immunomodulatory function of mesenchymal stem cells: mode of action and pathways. Ann N Y Acad Sci 1351:114-126. DOI:10.1111/nyas.12815

20. Ben-Mordechai T, Palevski D, Glucksam-Galnoy Y et al (2015) Targeting macrophage subsets for infarct repair. J Cardiovasc Pharmacol Ther 20:36-51. DOI:10.1177/1074248414534916

21. Lee JW, Fang X, Krasnodembskaya A et al (2011) Concise review: Mesenchymal stem cells for acute lung injury: role of paracrine soluble factors. Stem Cells 29:913-919. DOI:10.1002/stem.643

\section{Figures}

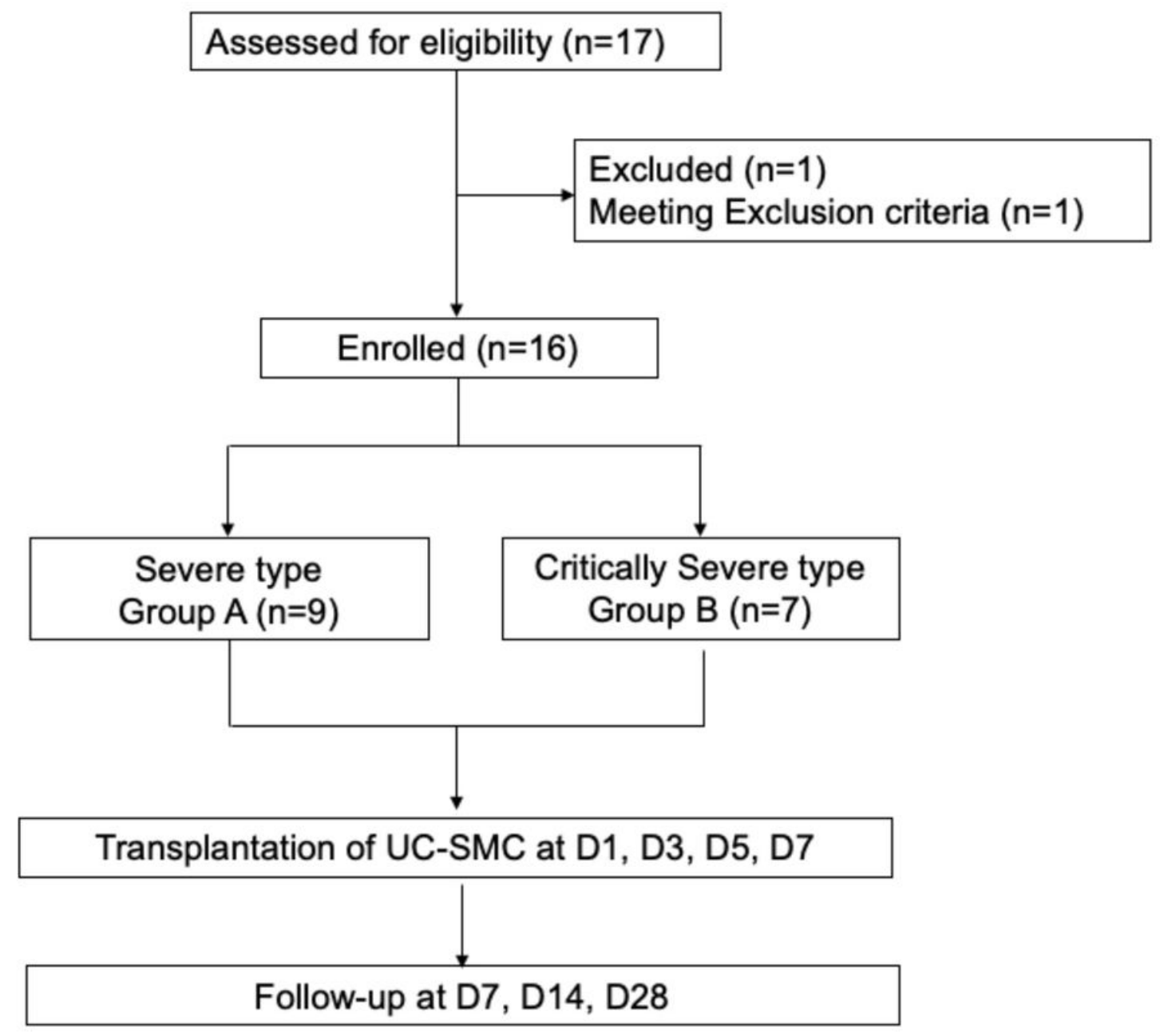

\section{Figure 1}

Flow diagram for patient enrollment, intervention, and follow-up. 


\section{Oxygenation index}

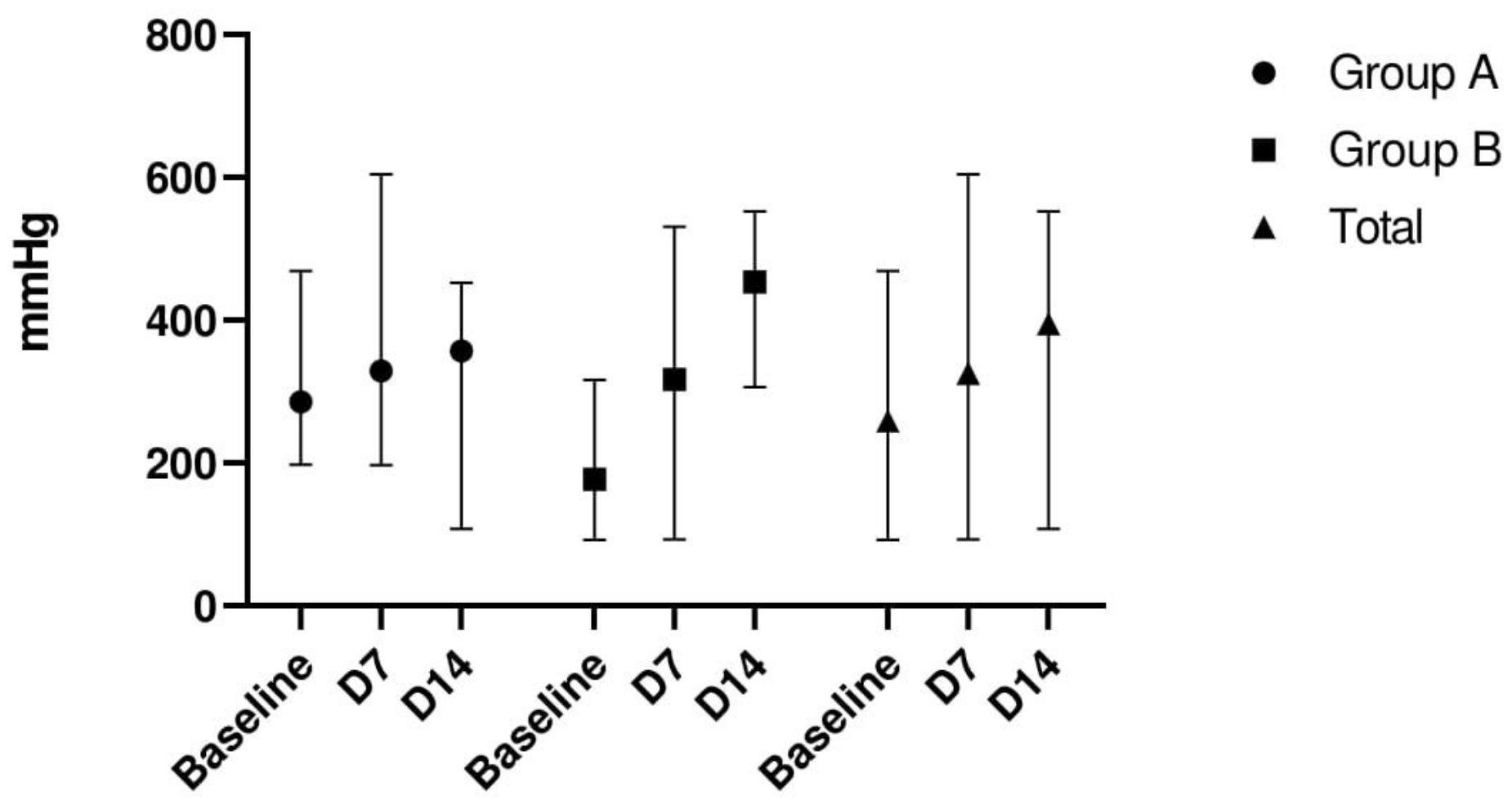

Figure 2

Oxygenation index at baseline (7 days before transplantation), D7, and D14 post treatment. Data was represented in median and range.

WBC

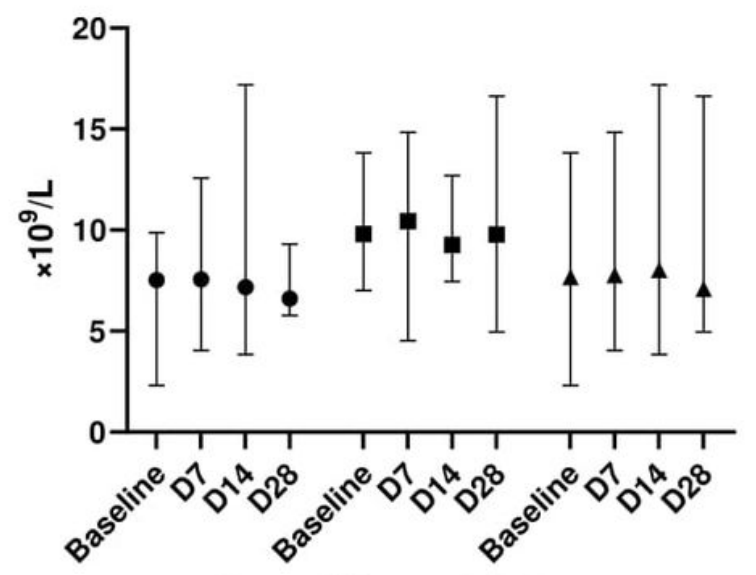

Normal Range : 3.5-9.5
Lymphocyte

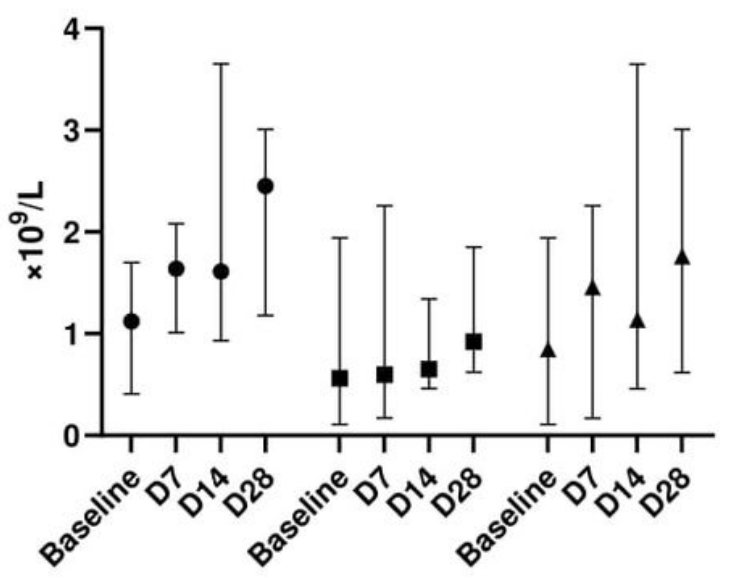

Normal Range: 1.1-3.2
- Group A

- Group B

- Total

Figure 3 
The white blood cell count and lymphocyte count at baseline (7 days before transplantation), D7, D14, and D28 post treatment.
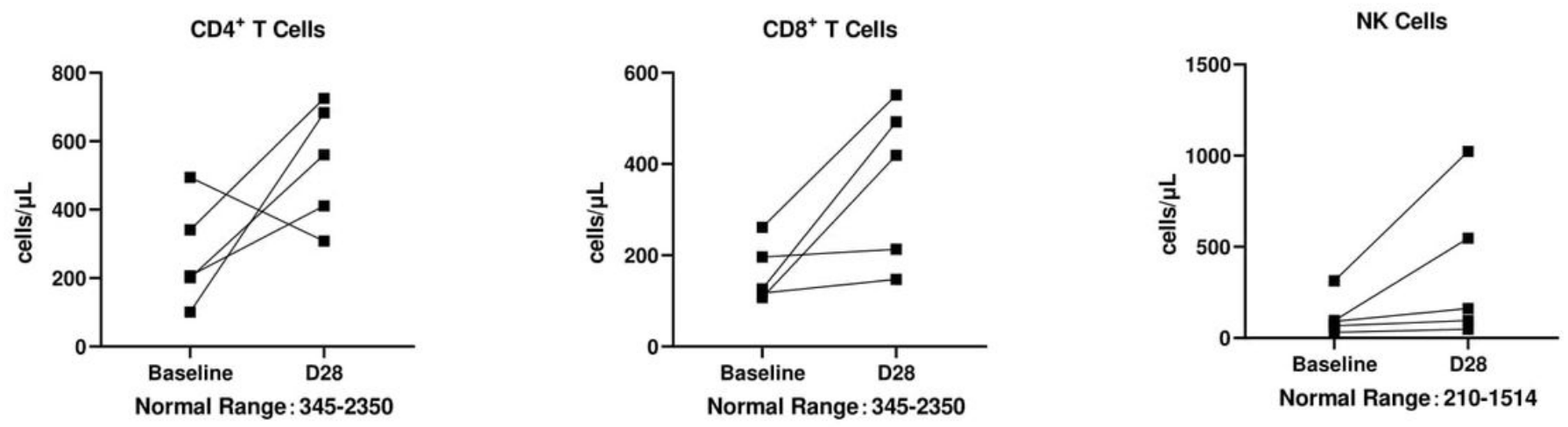

Figure 4

The lymphocyte subsets count at baseline (7 days before transplantation), and D28 post treatment.

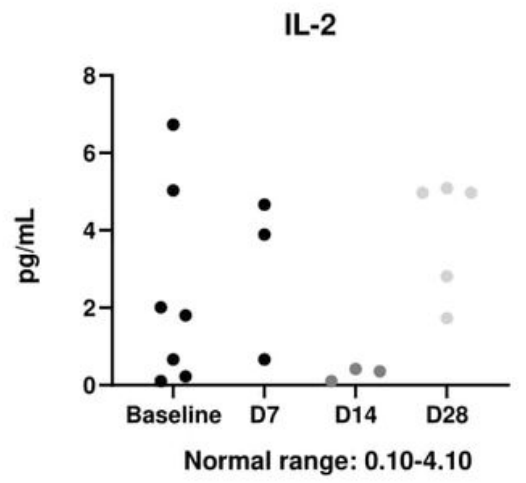

IL-10

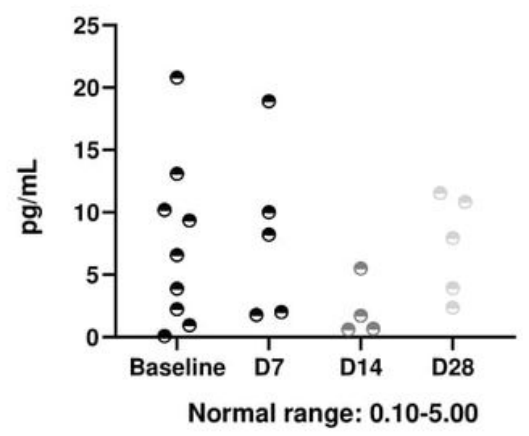

IL-4

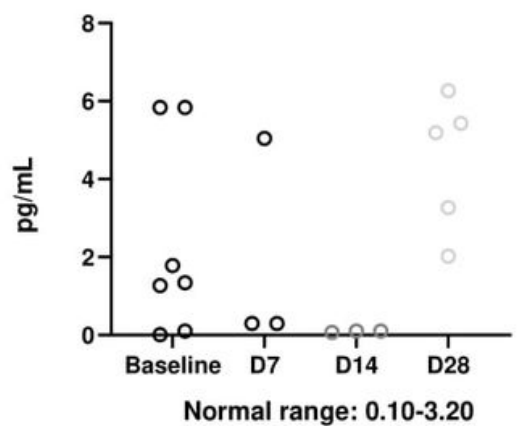

TNF- $\alpha$

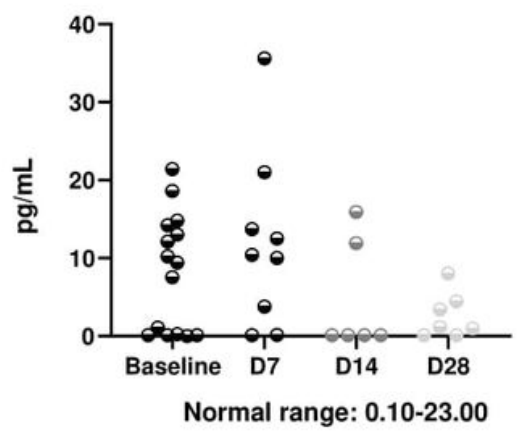

IL-6

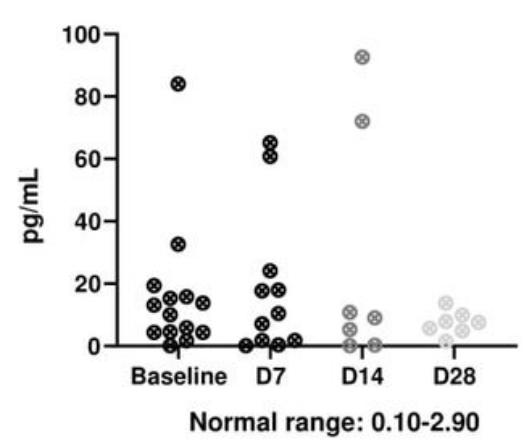

IFN-y

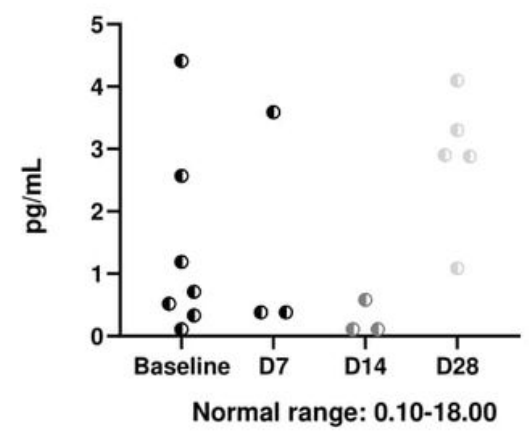

\section{Figure 5}

The cytokines at baseline (7 days before transplantation), D7, D14, and D28 post treatment. 


\section{CRP}

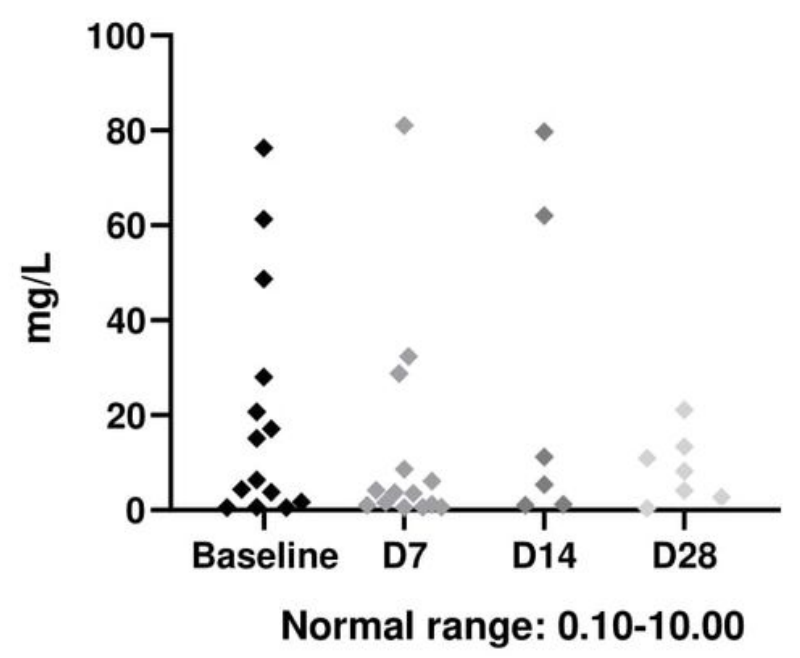

PCT

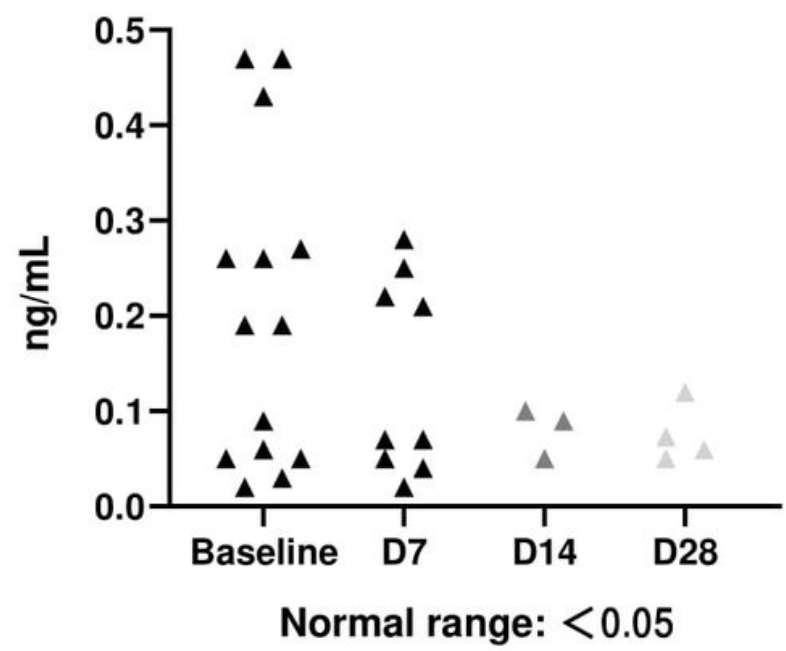

Figure 6

The $\mathrm{C}$ reaction protein (CRP) and procalcitonin (PCT) level at baseline (7 days before transplantation), D7, D14, and D28 post treatment. 